\title{
Synchronization in a System of Globally Coupled Oscillators with Time Delay
}

\author{
M. Y. Choi, H. J. Kim, and D. Kim \\ Department of Physics and Center for Theoretical Physics \\ Seoul National University, Seoul 151-742, Korea \\ H. Hong \\ Department of Physics Education and Center for Theoretical Physics \\ Seoul National University, Seoul 151-742, Korea
}

\begin{abstract}
We study the synchronization phenomena in a system of globally coupled oscillators with time delay in the coupling. The self-consistency equations for the order parameter are derived, which depend explicitly on the amount of delay. Analysis of these equations reveals that the system in general exhibits discontinuous transitions in addition to the usual continuous transition, between the incoherent state and a multitude of coherent states with different synchronization frequencies. In particular, the phase diagram is obtained on the plane of the coupling strength and the delay time, and ubiquity of multistability as well as suppression of the synchronization frequency is manifested. Numerical simulations are also performed to give consistent results.
\end{abstract}

PACS numbers: 05.45.+b, 02.30.Ks, 05.70.Fh, 87.10.+e

\section{INTRODUCTION}

When a large population of limit cycle oscillators with slightly different natural frequencies are coupled, they often come to oscillate with an identical frequency. Such collective synchronization phenomena have been observed in various oscillatory systems in physics, biology, chemistry, and other sciences [1, 迎, attracting much interest in recent years 5 11]. In the Kuramoto model for those oscillator systems, oscillators are coupled with each other via the interaction which depends on the phase difference between each pair [5]. It describes the emergence of phase coherence with the increase of the coupling strength, elucidating interesting connection between the collective synchronization and a phase transition.

Here, as in the usual dynamics of many-particle systems, sufficient attention has not been paid to the effects of time retardation in the oscillator system. In biological systems such as pacemaker cells and neurons, however, temporal delay is natural and the finite time interval required for the information transmission between two elements may be important [2,3]. Time delay in the interaction may modify drastically dynamic behavior of the system, such as stability and ergodicity [12]. In some types of a system of coupled oscillators, retarded interactions have been found to result in multistability and suppression of the collective frequency 13 16. In a system of two coupled oscillators, it has been found that the time delay induces a multitude of synchronized solutions. Namely, in the system with finite time delay, more than one stable solution are possible at given coupling strength. Among those, the most stable solution is the one with the largest synchronization frequency, as shown via the linear stability analysis [13]. Similar behaviors including frequency suppression and multistability have been observed in the neural network model, where peripheral oscillators with identical natural frequencies are coupled only with a central oscillator by forward and backward connections with time delay [14]. The two-dimensional system of identical oscillators with time-delayed nearest-neighbor coupling has also been considered to reveal similar frequency suppression [15]. The system of nonidentical oscillators with delayed interactions has been studied recently [17]: In the case of a Lorenzian distribution of natural frequencies with a nonzero mean, the stability boundary of the incoherent state has been obtained and coexistence of one or more coherent and/or incoherent states has been observed in appropriate regions [17. However, detailed behaviors such as frequency suppression and emergence of different coherent states have not been addressed fully.

This paper investigates in detail the effects of time delay in the interaction on collective synchronization of coupled oscillators with different natural frequencies. For this purpose, we derive the self-consistency equations for the order parameter and examine how the characteristic features of the collective synchronization change due to time delay. This reveals a multitude of coherent states with nonzero synchronization frequencies, each separated from the incoherent state by a discontinuous transition. In particular, we show that the system with a nonzero average frequency can be reduced to the system with the vanishing average natural frequency, which allows us to focus on the latter system. As in the system without delay, there exists the critical coupling strength, at which the system undergoes the usual 
continuous transition from the incoherent state to the coherent state displaying collective synchronization (with zero synchronization frequency). In addition, at higher values of the coupling strength (beyond the critical value), coherent states with larger synchronization frequencies also appear via discontinuous transitions. Thus coherent states with different synchronization frequencies in general coexist in the appropriate regions, leading to multistability. The synchronization frequency of the oscillators in a coherent state is observed to decrease with the delay time, which is similar to the result of other systems with time delay [14 16].

There are five sections in this paper: Section II presents the system of globally coupled oscillators with time delay, as a generalization of the Kuramoto model. The stationary probability distribution for the system is obtained, and the self-consistency equations for the order parameter are derived. Section III is devoted to the analysis of the selfconsistency equations, which reveals the characteristic behavior of the system as the coupling strength or the delay time is varied. In particular, the phase diagram is obtained on the plane of the coupling strength and the delay time, and ubiquity of multistability as well as suppression of the synchronization frequency is demonstrated. Numerical simulations are also performed and the results, which are in general consistent with the analytical ones, are presented in Sec. IV. Finally, Sec. V summarizes the main results, while some details of the calculations are presented in Appendices A and B.

\section{SYSTEM OF COUPLED OSCILLATORS WITH TIME DELAY} by

The set of equations of motion for $N$ coupled oscillators, each described by its phase $\phi_{i}(i=1,2, \cdots, N)$, is given

$$
\dot{\phi}_{i}(t)=\omega_{i}-\frac{K}{N} \sum_{j}^{\prime} \sin \left[\phi_{i}(t)-\phi_{j}(t-\tau)\right],
$$

where the prime restricts the summation such that $j \neq i$. The first term on the right-hand side represents the natural frequency of the $i$ th oscillator, which is distributed according to the distribution function $g(\omega)$. Here $g(\omega)$ is assumed to be smooth and symmetric about $\omega_{0}$, which may be taken to be zero without loss of generality (see below), and also to be concave at $\omega=0$, i.e., $g^{\prime \prime}(0)<0$. The second term denotes the global coupling of strength $K / N$ between oscillators, with time delay, indicating that each oscillator interacts with other oscillators only after the retardation time $\tau$. Without time delay, Eq. (1) exactly reduces to the Kuramoto model.

In order to describe collective synchronization of such an $N$ oscillator system, we define the complex order parameter, whose amplitude represents the degree of synchronization, to be

$$
\Psi \equiv \frac{1}{N} \sum_{j=1}^{N} e^{i \phi_{j}}=\Delta e^{i \theta}
$$

Here it is convenient to introduce new variables $\psi_{i}$ defined by $\psi_{i} \equiv \phi_{i}-\Omega t$, where $\Omega$ is a constant. Note the existence of physical invariance due to the rotational symmetry of the total system. In terms of the new variables, Eq. (11) reads

$$
\dot{\psi}_{i}=\tilde{\omega}_{i}-\frac{K}{N} \sum_{j}^{\prime} \sin \left[\psi_{i}(t)-\psi_{j}(t-\tau)+\Omega \tau\right]
$$

where $\tilde{\omega}_{i} \equiv \omega_{i}-\Omega$. Multiplying Eq. (2) by $e^{-i \Omega t}$, we also obtain the corresponding order parameter for the new variables

$$
\tilde{\Psi} \equiv \frac{1}{N} \sum_{j=1}^{N} e^{i \psi_{j}}=\Delta e^{i \tilde{\theta}}
$$

where $\tilde{\theta} \equiv \theta-\Omega t$. Incidentally, the order parameter defined in Eq. (4) allows us to reduce Eq. (3) into a single decoupled equation with time delay

$$
\dot{\psi}_{i}=\tilde{\omega}_{i}-K \Delta \sin \left(\psi_{i}-\theta_{0}\right)
$$

where $\theta_{0} \equiv \tilde{\theta}-\Omega \tau$. Although $\Delta, \Omega$, and $\theta_{0}$ depend on the delay time $\tau$, they are assumed to be independent of time $t$, which is possible due to the symmetry. Considering the relation between the old order parameter and the new one 


$$
\Psi=\tilde{\Psi} e^{i \Omega t}
$$

we understand that the collective synchronization can be described in terms of a giant oscillator rotating with the frequency $\Omega$ which is in general nonzero. For finite delay time, there exists a multitude of synchronized solutions with nonzero values of $\Omega$; this is in contrast to system without delay, where the rotational symmetry of the system allows us to set $\Omega=0$.

Instead of Eq. (5), which may be regarded as a Langevin equation without noise, one may resort to the corresponding Fokker-Planck equation for the probability distribution $P(\psi, t)$ at zero temperature [18]:

$$
\frac{\partial}{\partial t} P(\psi, t)=\frac{\partial}{\partial \psi}\left[K \Delta \sin \left(\psi-\theta_{0}\right)-\tilde{\omega}\right] P(\psi, t)
$$

The order parameter given by Eq. (4) then obtains the form

$$
\begin{aligned}
\Delta e^{i \tilde{\theta}} & =\frac{1}{N} \sum_{j=1}^{N} e^{i \psi_{j}} \\
& =\int_{-\infty}^{\infty} d \tilde{\omega} g(\tilde{\omega}+\Omega)\left\langle e^{i \psi}\right\rangle_{t-\tau ; \tilde{\omega}}
\end{aligned}
$$

where $\tilde{\omega}=\omega-\Omega$ has been noted, and the average of $e^{i \psi}$ is to be taken over the distribution $P(\psi, t-\tau)$ of Eq. (7) with given $\tilde{\omega}$ : $\left\langle e^{i \psi}\right\rangle_{t-\tau ; \tilde{\omega}} \equiv \int_{0}^{2 \pi} d \psi P(\psi, t-\tau) e^{i \psi}$.

In the stationary state, we take the average over the stationary distribution $P^{(0)}(\psi ; \tilde{\omega})$ of Eq. (『). With the stationary solution

$$
P^{(0)}(\psi ; \tilde{\omega})= \begin{cases}\delta\left[\psi-\theta_{0}-\sin ^{-1}(\tilde{\omega} / K \Delta)\right] & \text { for }|\omega| \leq K \Delta \\ \frac{\sqrt{\tilde{\omega}^{2}-(K \Delta)^{2}}}{2 \pi\left|\tilde{\omega}-K \Delta \sin \left(\psi-\theta_{0}\right)\right|} & \text { otherwise, }\end{cases}
$$

it is easy to compute the average:

$$
\begin{aligned}
\left\langle e^{i \psi}\right\rangle_{\tilde{\omega}} & \equiv \int_{0}^{2 \pi} d \psi P^{(0)}(\psi ; \tilde{\omega}) e^{i \psi} \\
& =e^{i \theta_{0}} \begin{cases}i(\tilde{\omega} / K \Delta)-i \sqrt{(\tilde{\omega} / K \Delta)^{2}-1}, & \tilde{\omega}>K \Delta \\
i(\tilde{\omega} / K \Delta)+\sqrt{1-(\tilde{\omega} / K \Delta)^{2}}, & -K \Delta \leq \tilde{\omega} \leq K \Delta \\
i(\tilde{\omega} / K \Delta)+i \sqrt{(\tilde{\omega} / K \Delta)^{2}-1}, & \tilde{\omega}<-K \Delta .\end{cases}
\end{aligned}
$$

It is thus natural to divide the system into two groups: one satisfying $|\tilde{\omega}| \leq K \Delta$, which is called the synchronization group, and the other $|\tilde{\omega}|>K \Delta$, the desynchronization group. Accordingly, we write

$$
\Delta=\Delta_{s}+\Delta_{d}
$$

where $\Delta_{s / d}$ is the contribution from the synchronization/desynchronization group to the order parameter. The contribution from the synchronization group is given by

$$
\Delta_{s} e^{i \Omega \tau}=K \Delta \int_{-1}^{1} d x g(\Omega+K \Delta x)\left[\sqrt{1-x^{2}}+i x\right],
$$

where $x \equiv \tilde{\omega} / K \Delta$. Separating Eq. (12) into the real and imaginary parts, we obtain the two coupled nonlinear equations

$$
\begin{aligned}
& \Delta_{s} \cos \Omega \tau=K \Delta \int_{-1}^{1} d x g(\Omega+K \Delta x) \sqrt{1-x^{2}}, \\
& \Delta_{s} \sin \Omega \tau=K \Delta \int_{-1}^{1} d x g(\Omega+K \Delta x) x .
\end{aligned}
$$

Similarly, the desynchronization group leads to the equation 


$$
\begin{aligned}
\Delta_{d} e^{i \Omega \tau}= & i K \Delta \int_{1}^{\infty} d x g(\Omega+K \Delta x)\left(x-\sqrt{x^{2}-1}\right) \\
& +i K \Delta \int_{-\infty}^{-1} d x g(\Omega+K \Delta x)\left(x+\sqrt{x^{2}-1}\right)
\end{aligned}
$$

or

$$
\begin{aligned}
\Delta_{d} \cos \Omega \tau=0, & \\
\Delta_{d} \sin \Omega \tau=K \Delta & {\left[\int_{1}^{\infty} d x g(\Omega+K \Delta x)\left(x-\sqrt{x^{2}-1}\right)\right.} \\
& \left.+\int_{-\infty}^{-1} d x g(\Omega+K \Delta x)\left(x+\sqrt{x^{2}-1}\right)\right] .
\end{aligned}
$$

Note that, unlike the Kuramoto model, the imaginary parts of Eqs. (12) and (14) do not vanish, which arises from the fact that the nonzero collective frequency due to time delay breaks the symmetry of the integration interval of the distribution $g(\omega)$. It is obvious in Eq. (15) that the contribution from the desynchronization group vanishes in the absence of time delay $(\tau=0)$. Recalling that in the presence of time delay the total order parameter is given by the sum of the two contributions, one from the synchronization group and the other from the desynchronization group, we finally obtain the self-consistency equations from Eqs. (13) and (15):

$$
\begin{aligned}
& \Delta \cos \Omega \tau=K \Delta \int_{-1}^{1} d x g(\Omega+K \Delta x) \sqrt{1-x^{2}}, \\
& \Delta \sin \Omega \tau=K \Delta\left[\int_{-1}^{1} d x g(\Omega+K \Delta x) x+\int_{1}^{\infty} d x g(\Omega+K \Delta x)\left(x-\sqrt{x^{2}-1}\right)\right. \\
& \left.+\int_{-\infty}^{-1} d x g(\Omega+K \Delta x)\left(x+\sqrt{x^{2}-1}\right)\right] .
\end{aligned}
$$

When the average natural frequency is not zero $\left(\omega_{0} \neq 0\right)$, we define the variables $\psi_{i} \equiv \phi_{i}-\left(\Omega+\omega_{0}\right) t$, and obtain exactly Eq. (16) except for $\Omega$ replaced by $\tilde{\Omega} \equiv \Omega+\omega_{0}$. For example, the first equation is given by

$$
\Delta \cos \tilde{\Omega} \tilde{\tau}=K \Delta \int_{-1}^{1} d x \tilde{g}(\tilde{\Omega}+K \Delta x) \sqrt{1-x^{2}}
$$

where $\tilde{\tau}$ is the delay time and the distribution $\tilde{g}(\omega)$ is symmetric about $\omega=\omega_{0}$. Since the distribution $g(\omega) \equiv \tilde{g}\left(\omega+\omega_{0}\right)$ is symmetric about $\omega=0$, we rewrite the above equation in the form

$$
\Delta \cos \left[\left(\Omega+\omega_{0}\right) \tilde{\tau}\right]=K \Delta \int_{-1}^{1} d x g(\Omega+K \Delta x) \sqrt{1-x^{2}},
$$

which, with the identification $\left(\Omega+\omega_{0}\right) \tilde{\tau} \equiv \Omega \tau$, just reproduces the first equation in Eq. (16). Accordingly, the behavior of the system with $\omega_{0} \neq 0$, which has been considered in Ref. [17], can be obtained from that of the system with $\omega_{0}=0$ via appropriate rescaling of parameters.

\section{ANALYSIS OF THE SELF-CONSISTENCY EQUATIONS}

The non-vanishing imaginary part of the self-consistency equation given by Eq. (16), arising from time delay, leads to a variety of behaviors which are not displayed by the system without delay. In this section, we solve the two coupled equations in Eq. (16) to obtain the synchronization frequency $\Omega$ and the order parameter $\Delta$. We take the Gaussian distribution with zero mean and unit variance for the natural frequencies: $g(\omega)=(2 \pi)^{-1 / 2} e^{-\omega^{2} / 2}$, and first compute numerically the synchronization frequency and the order parameter for various values of the coupling strength $K$ and the delay time $\tau$.

Figure 1 exhibits the dependence of the synchronization frequency on $K$ and $\tau$, which manifests multistability. At small values of $\tau$, only the nontrivial solution $(\Delta \neq 0)$ with $\Omega=0$ appears for $K>K_{c}(\approx 1.596)$, as in the system without delay. For large $\tau$, on the other hand, solutions with $\Omega \neq 0$ also emerge as $K$ is increased further. In Fig. 1(a), where the synchronization frequency is plotted as a function of the delay time $\tau$ at $K=10$, it is also 
observed that the synchronization frequency is suppressed as time delay is increased. This is expected since the delay tends to disturb synchronization 14. In Fig. 1(b), we plot the synchronization frequency as a function of the coupling strength at $\tau=5$. It shows that at given values of $\tau$ the synchronization frequency $\Omega$ depends rather weakly on $K$ after synchronization sets in. Among those solutions at given coupling strength $K$, the most stable solution is the one with the largest value of $\Omega$ [13] although the basin of attraction in general shrinks with $\Omega$.

The phase boundaries separating the coherent states $(\Delta \neq 0)$ with various synchronization frequencies from the incoherent state $(\Delta=0)$ are shown in Fig. 2, where data have been taken with the step width $\delta \tau=0.06$. Below the lowest boundary, which is the straight line $K=K_{c} \approx 1.596$, only the incoherent state exists; above it, the coherent state with $\Omega=0$ also exist. Similarly, above each boundary in Fig. 2(a), a new additional coherent state with a larger synchronization frequency emerges. Note here that the region of the existence of coherent states constitutes two-dimensional (semi-infinite) surfaces in the three-dimensional $(K, \tau, \Omega)$ space. Whereas Figs. 1(a) and (b) may be regarded as the cross-sections of these surfaces at given values of $K$ and of $\tau$, respectively, Fig. 2(a) represents the projection of the boundaries of these surfaces onto the $K-\tau$ plane. In Fig. 2(b) the curves of Fig. 2(a) are redrawn, with the horizontal axis rescaled: $\tilde{\tau}$ in (b) corresponds to $\Omega \tau /(\Omega+3)$ in (a). In this new scale of the horizontal axis, unlike in Fig. 2(a), the boundaries intersect with each other, and only the envelope consisting of the curve segments with lowest values of $K$ at given $\tilde{\tau}$ is displayed in Fig. 2(b). According to the discussion in Sec. II, Fig. 2(b) describes the phase boundary below which only the incoherent state exists in the system with $\omega_{0}=3$. Above the boundary, the coherent state with the appropriate (nonzero) synchronization frequency, depending on $\tilde{\tau}$, appears and can coexist with the incoherent state. Note that the lowest boundary ( $\left.K=K_{c} \approx 1.596\right)$ in Fig. 2(a) has no counterpart in Fig. 2(b) since the zero synchronization frequency $(\Omega=0)$ corresponds to $\tilde{\tau}=0$. Similar boundaries have been obtained through numerical simulations for the Lorenzian as well as the delta-function distributions [17]. It is of interest to compare Fig. 2(b) with Fig. 4 in Ref. [17], which indicates that the Gaussian distribution leads to smoother stability boundaries than the Lorenzian distribution.

In Fig. 3, the obtained order parameter $\Delta$ is depicted as a function of $K$ at $\tau=5$. Each line describes the transition for each synchronization frequency, and the critical coupling strength $K_{c}$ is shown to increase for the transition with larger $\Omega$. For example, the leftmost curve, which corresponds to $\Omega=0$, gives $K_{c} \approx 1.596$, whereas the next one, corresponding to $\Omega \approx 1.09$, gives $K_{c} \approx 1.97$. Note that as $K$ approaches $K_{c}(\approx 1.596)$, the order parameter with $\Omega=0$ decreases continuously to zero, indicating that the leftmost curve describes a continuous transition at $K_{c}$. On the other hand, the rest of the curves with $\Omega>0$ apparently display jumps in the order parameter, indicating discontinuous transitions. Accordingly, whereas the lowest boundary in Fig. 2(a) describes a continuous transition, the others as well as the boundary in Fig. 2(b) correspond to discontinuous transitions.

To understand the nature of these transitions analytically, we assume $K \Delta \ll 1$ near the transition to the coherent state and expand Eq. (16) to the order of $(K \Delta)^{3}$, together with $\Omega$ also expanded accordingly:

$$
\Omega \approx \Omega_{0}+\Omega_{1} K \Delta+\Omega_{2}(K \Delta)^{2} .
$$

We investigate two regimes, $\Omega \ll K \Delta$ and $\Omega \gg K \Delta$, which exhibit phase transitions of different types with each other, still taking the Gaussian distribution with zero mean and unit variance for $g(\omega)$.

For $\Omega \ll K \Delta(\ll 1)$, the self-consistency equation for the order parameter takes the form

$$
\Delta=a_{1} K \Delta+b_{1}(K \Delta)^{2}+c_{1}(K \Delta)^{3}+\mathcal{O}(K \Delta)^{4},
$$

where the coefficients $a_{1}, b_{1}$, and $c_{1}$ depend on $\Omega_{0}, \Omega_{1}$, and $\Omega_{2}$ defined in Eq. (19). Their specific forms as well as the details of the calculation are given in Appendix A. Since the condition $\Omega \ll K \Delta$ implies $\Omega_{0} \ll 1$, we need to consider Eq. A.3 only for the range $-\pi / 2<\tan ^{-1}\left(\sqrt{2 / \pi} \Omega_{0} e^{\Omega_{0}^{2} / 2}\right)<\pi / 2$. It is then obvious that the desired solution of Eq. (A.3) is simply $\Omega_{0}=\Omega_{1}=\Omega_{2}=0$, regardless of $\tau$. Inserting these values into Eq. (A.5), we obtain the values of the coefficients: $a_{1}=0.626, b_{1}=0$, and $c_{1}=-0.078$. Figure 4 illustrates the graphical solution of Eq. (20), displaying $f(\Delta) \equiv\left(a_{1} K-1\right) \Delta+b_{1}(K \Delta)^{2}+c_{1}(K \Delta)^{3}$ versus $\Delta$ for $b_{1}=0$. Note that the critical coupling strength is given by $K_{c}\left(\equiv a_{1}{ }^{-1}\right) \approx 1.595$, which indeed agrees perfectly with the numerical value given by the leftmost curve in Fig. 3. It is thus concluded that the system displays a continuous phase transition with $\Omega=0$, which is consistent with the result of the Kuramoto model.

In the opposite case of $\Omega \gg K \Delta(\ll 1)$, we can still obtain the self-consistency equation for $\Delta$ up to the order of $(K \Delta)^{3}$, in a manner similar to that for the previous small- $\Omega$ case:

$$
\Delta=a_{2} K \Delta+b_{2}(K \Delta)^{2}+c_{2}(K \Delta)^{3}+\mathcal{O}(K \Delta)^{4},
$$

where the coefficients $a_{2}, b_{2}$, and $c_{2}$ again depend on $\Omega_{0}, \Omega_{1}$, and $\Omega_{2}$ (see Appendix B). In this case, we need to obtain larger solutions, considering the regions $(n+1 / 2) \pi<\tan ^{-1}\left(\sqrt{2 / \pi} \Omega_{0} e^{\Omega_{0}^{2} / 2}\right)<(n+3 / 2) \pi$ with non-negative integer 
$n$. Interestingly, this in general yields nonzero values of $\Omega_{0}$ and accordingly, nonzero values of $b_{2}$, with which Eq. (21) displays a jump in $\Delta$ at $K=-4 c_{2}\left(b_{2}{ }^{2}-4 c_{2} a_{2}\right)^{-1}$, thus indicating a discontinuous transition [9]. Such discontinuous transitions are ubiquitous for regions with higher values of $n$. Namely, the system with delay is in general characterized by nonzero values of the synchronization frequency together with discontinuous transitions, which is consistent with the numerical results displayed in Fig. 3. There the jumps in $\Delta$ displayed by the curves with $\Omega>0$, associated with discontinuous transitions, may invalidate the assumption $K \Delta \ll 1$, and the expansion in Eq. (21) is not expected to yield quantitatively accurate results. Nevertheless the appearance of such discontinuous transitions has been revealed by the above expansion, which is concluded to give a qualitatively correct description of the nature of transitions.

For more accurate results, we investigate these transition phenomena by examining in detail the behaviors of the solutions of the self-consistency equations. Note that $\Delta=0$ is always a solution of Eq. (16) for all values of $\Omega$. To seek for other solutions, we divide Eq. (16) by $\Delta$ and obtain

$$
\begin{aligned}
K^{-1} \cos \Omega \tau= & \int_{-1}^{1} d x g(\Omega+K \Delta x) \sqrt{1-x^{2}} \\
K^{-1} \sin \Omega \tau= & \int_{-\infty}^{\infty} d x g(\Omega+K \Delta x) x-\int_{1}^{\infty} d x g(\Omega+K \Delta x) \sqrt{x^{2}-1} \\
& \quad+\int_{-\infty}^{-1} d x g(\Omega+K \Delta x) \sqrt{x^{2}-1},
\end{aligned}
$$

which may be computed numerically. The resulting values of $\Omega$ versus $\Delta$ are plotted in Figs. 5-7 for $\tau=5$. The solid and the dotted lines represent solutions of the first (real part) and the second (imaginary part) equations of Eq. (22), respectively. In each figure, the point where the two lines meet with each other provides the synchronization frequency $\Omega$ and the order parameter $\Delta$. Figure 5 (a) shows the absence of a meeting point for $K=1.58$, which implies that synchronization does not set in yet. In contrast, the meeting of the solid and dotted lines is obvious for $K=1.60$ shown in (c); (b) reveals a continuous transition (for $\Omega=0$ ) at $K=K_{c} \approx 1.596$, which coincides with the previous result. The value of $\Delta$ grows continuously as $K$ is increased beyond $K_{c}$ (see Fig. 6). When $K$ reaches the value 1.97, as displayed in Fig. 6(b), there emerges via a tangent bifurcation an additional meeting point at finite values of $\Delta(\approx 0.08)$ and $\Omega(\approx 1.09)$, giving rise to a discontinuous transition, in agreement with the result shown in Fig. 3. As the coupling strength is increased further, there appear two meeting points, giving two values of the order parameter for the pair of the lines (i.e., with almost the same value of $\Omega$ ), as shown in Fig. 6(c). Such a tangent bifurcation in general produces a pair of stable and unstable solutions; here the solution with the smaller value of $\Delta$, decreasing with $K$, should be unstable. Figure 7 (c) shows that the unstable solution becomes null $(\Delta=0)$ at $\Omega \approx 1.257$ as $K$ approaches $K_{0} \approx 3.515$. Figure 7 also reveals the occurrence of the third transition at $K_{c} \approx 3.46$, which is of the same nature as the second.

The values of $K_{c} \approx 1.596$ and $K_{0} \approx 3.515$ can also be obtained analytically since they are given by the solutions of Eq. (22) in the limit $\Delta \rightarrow 0$. In this limit, the right-hand side of the second equation vanishes, yielding $\Omega=n \pi / \tau$ with $n$ integer. The first one, which reduces to $K^{-1} \cos \Omega \tau=(\pi / 2) g(\Omega)$, then gives

$$
K=\frac{2}{\pi g(2 n \pi / \tau)},
$$

where it has been noted that $K>0$. Taking $n=0$ and $n=1$ in Eq. (23), where $g(\omega)$ is given by the Gaussian distribution with unit variance, we obtain $K=K_{c}=\sqrt{8 / \pi} \approx 1.596$ (with $\Omega=0$ ) and $K=K_{0}=\sqrt{8 / \pi} e^{2(\pi / \tau)^{2}} \approx$ 3.515 (with $\Omega=2 \pi / \tau \approx 1.257$ ) for $\tau=5$, respectively.

To examine how the stability changes at these bifurcations, we now consider a small perturbation from the incoherent state, for which the stationary distribution in Eq. (9) is simply given by $1 / 2 \pi$, and write

$$
P(\psi, t)=\frac{1}{2 \pi}+\epsilon \eta(\psi, t),
$$

where $\epsilon \ll 1$. Upon substitution into Eq. (7) and with $\Delta=\Delta_{1} \epsilon+\mathcal{O}\left(\epsilon^{2}\right)$, we obtain, to the lowest order in $\epsilon$,

$$
\frac{\partial \eta}{\partial t}=-\tilde{\omega} \frac{\partial \eta}{\partial \psi}+\frac{K}{2 \pi} \Delta_{1} \cos \left(\psi-\theta_{0}\right)
$$

and seek solutions of the form

$$
\eta(\psi, t)=c(t ; \tilde{\omega}) e^{i \psi}+c^{*}(t ; \tilde{\omega}) e^{-i \psi}
$$


where higher harmonics have been neglected. Equations (25) and (26), together with Eq. (8), lead to the amplitude equation for $c(t ; \tilde{\omega})$ :

$$
\frac{\partial c(t, \tilde{\omega})}{\partial t}=-i \tilde{\omega} c(t, \tilde{\omega})+\frac{K}{2} e^{i \Omega \tau} \int_{-\infty}^{\infty} d \tilde{\omega} g(\tilde{\omega}+\Omega) c(t-\tau, \tilde{\omega}),
$$

which in general possesses both discrete and continuous spectra. To find out the discrete spectrum, we put

$$
c(t ; \tilde{\omega})=b(\tilde{\omega}) e^{\lambda t},
$$

where the eigenvalue $\lambda$ is independent of $\tilde{\omega}$, and obtain the equation

$$
e^{-(\lambda-i \Omega) \tau} \frac{K}{2} \int_{-\infty}^{\infty} d \omega \frac{g(\omega)}{\lambda+i(\omega-\Omega)}=1
$$

which has been examined for a Lorenzian distribution [17].

Here we investigate Eq. (29) for a Gaussian distribution. The stability of the incoherent state depends on whether all roots of Eq. (29) possess negative real parts, i.e., $\operatorname{Re} \lambda<0$. This is the case for $K$ less than $K_{s}$, where the incoherent state is neutrally stable (since the continuous spectrum is pure imaginary). Beyond $K_{s}$, there appears an eigenvalue with a positive real part, giving rise to instability. The value of $K_{s}$ can be computed from Eq. (29) with $\operatorname{Re} \lambda=0$ imposed; this yields the coupled equations for $K_{s}$ and $\operatorname{Im} \lambda$

$$
\begin{aligned}
\cos \gamma \tau & =K_{s} \sqrt{\frac{\pi}{8}} e^{-\gamma^{2} / 2} \\
\sin \gamma \tau & =-\frac{K_{s}}{2} \gamma e^{-\gamma^{2} / 2} \sum_{k=0}^{\infty} \frac{\gamma^{2 k}}{2^{k}(2 k+1) k !}
\end{aligned}
$$

where $\gamma \equiv \Omega-\operatorname{Im} \lambda$. Unlike the system without delay, Eq. (30) has an infinite number of solutions, among which the lowest value of $K_{s}$ should be taken. It is obvious that $\gamma=0$ is the desired solution (regardless of time delay), leading to the lowest value $K_{s}=\sqrt{8 / \pi} \approx 1.596$. Note also that this value of $K_{s}$ coincides exactly with that of $K_{c}$, implying that the incoherent state becomes unstable simultaneously with the appearance of the (stable) coherent state with $\Omega=0$.

These results reveal that the order parameter exhibits a supercritical bifurcation at $K=K_{c}$ along the leftmost curve $(\Omega=0)$ in Fig. 3. Namely, the emergence of a nontrivial solution $(\Delta>0)$ is accompanied by the loss of stability of the null solution $(\Delta=0)$ at $K_{c}(\Omega=0) \approx 1.596$. For the rest $(\Omega>0)$, on the other hand, the unstable solution, generated together with the stable one by a tangent bifurcation at $K_{c}(\Omega)$, decreases as $K$ is raised further and vanishes to zero at a larger value, $K=K_{0}(\Omega)$. For example, the unstable solution for $\Omega \approx 1.09$, emerging at $K \approx 1.97$, decreases to zero at $K \approx 3.515$ [see Fig. 7 (c)]. It is thus concluded that for $\Omega>0$ the bifurcation at $K_{0}$ is subcritical: Between $K_{c}$ and $K_{0}$ there exist an unstable coherent state in addition to the stable coherent states (and the incoherent one) although the unstable states have not been displayed in Fig. 3.

The general features of the synchronization behavior obtained here are similar to those in Ref. [17], and it is thus concluded that the difference in the distribution of natural frequencies does not change results qualitatively. On the other hand, we have examined additional interesting phenomena such as frequency suppression and details of multistability. In particular, unlike in the system with $\omega_{0} \neq 0$ considered mostly in Ref. 17], here the phase boundaries with different values of the synchronization frequency do not intersect with each other on the $K-\tau$ plane. [Compare Fig. 2(a) and (b).] Accordingly, the system with $\omega_{0}=0$ does not undergo a discontinuous transition directly from the stable incoherent state and the coherent one with a nonzero synchronization frequency, and the associated hysteresis may not be observed. Further, in order to confirm these results, we have also performed numerical simulations, the results of which are presented in the next section.

\section{NUMERICAL SIMULATIONS}

We have studied directly the equations of motion given by Eq. (1) via numerical simulations. The globally coupled system of size $N=5000$, where natural frequencies are distributed according to the Gaussian distribution with unit variance, has been considered, and the Euler method with discrete time steps of $\delta t=0.01$ has been employed. At each run, we have discarded the first $10^{5}$ time steps per oscillator to eliminate transient effects and taken the next $10^{5}$ time steps per oscillator to investigate synchronized solutions. Finally, independent runs with 30 different realizations 
of the natural frequency distribution and initial conditions have been performed, over which the averages have been taken. In the simulations, the synchronization frequency is given by the average phase speed, i.e., the average rate of the phase change, and the obtained data at the coupling strength $K=10$ are represented by crosses in Fig. 8(a). Note that both the incoherent state and the coherent one are found to be stable at the same value of $\tau$, indicating multistability; frequency suppression with increasing delay is also manifested. For comparison, the results shown in Fig. 1(a), obtained from Eq. (16), are also displayed, and perfect agreement is observed. Notice here that the basin of attraction shrinks rapidly with the synchronization frequency $\Omega$, which makes it quite difficult in numerical simulations to find the coherent-state solutions with large values of $\Omega$. Figure $8(\mathrm{~b})$ shows the behavior of the order parameter $\Delta$ as a function of the coupling strength $K$ for $\tau=0$ (plus signs) and $\tau=5$ (crosses). In both cases the system displays a continuous transition to the coherent state (with zero synchronization frequency). Slight suppression of synchronization by time delay can be observed. The error bars have been estimated by the standard deviation and the lines are guides to the eye. To make comparison of the analytical results obtained from Eq. (16) and the simulation results, we have also included in Fig. 8(b) the analytical results for $\tau=5$, which are represented by the solid line. Good overall agreement between the two can be observed.

\section{SUMMARY}

We have studied analytically and numerically the collective synchronization phenomena in a set of globally coupled oscillators with time retarded interaction. In order to understand the effects of time delay on the synchronization, we have derived the self-consistency equations for the order parameter, which describe synchronization in the system. The detailed analysis of the self-consistency equations has revealed a multitude of coherent states with nonzero synchronization frequencies, each separated from the incoherent state by a discontinuous transition. At the critical coupling strength, the system exhibits the usual continuous transition from the incoherent state to the coherent one, displaying collective synchronization with zero synchronization frequency. As the coupling strength is increased further, coherent states with larger synchronization frequencies have also been shown to appear via discontinuous transitions from the incoherent state. Thus a multitude of coherent states with different synchronization frequencies have been found to coexist in the appropriate regions, leading to multistability. The synchronization frequency of the oscillators in a coherent state has been observed to decrease with the delay time.

To confirm the analytical results, we have also performed numerical simulations, the results of which indeed display multistability and suppression of the synchronization frequency. For detailed comparison, however, one should search the solution space extensively, with varying initial conditions, to obtain solutions with various values of the synchronization frequency. This requires more extensive simulations, which is left for future study. Finally, one may also include stochastic noise in the system and study its effects on synchronization behavior. In particular, the interplay between the external driving and noise poses the possibility of stochastic resonance 19], and it is of interest to examine how the collective synchronization together with the time delay affects the possible resonance phenomena.

\section{ACKNOWLEDGMENTS}

We thank G. S. Jeon, M.-S. Choi, and K. Park for illuminating discussions and C. W. Kim for the hospitality during our stay at Korea Institute for Advanced Study, where part of this work was accomplished. This work was supported in part by the SNU Research Fund, by the Korea Research Foundation, and by the Korea Science and Engineering Foundation.

\section{APPENDIX A}

In the case $\Omega \ll K \Delta(\ll 1)$, we approximate the integral appearing in Eq. (16):

$$
\begin{aligned}
& \int_{1}^{\infty} d x g(\Omega+K \Delta x)\left(x-\sqrt{x^{2}-1}\right)+\int_{-\infty}^{-1} d x g(\Omega+K \Delta x)\left(x+\sqrt{x^{2}-1}\right) \\
& =\int_{1}^{\infty} d x[g(\Omega+K \Delta x)-g(\Omega-K \Delta x)]\left(x-\sqrt{x^{2}-1}\right) \\
& \approx \int_{1}^{\infty} d x 2 \Omega g^{\prime}(K \Delta x)\left(x-\sqrt{x^{2}-1}\right)
\end{aligned}
$$


and expand Eq. (16) to the order $(K \Delta)^{3}$. This yields

$$
\begin{aligned}
& \Delta \cos \left[\left(\Omega_{0}+\Omega_{1}(K \Delta)+\Omega_{2}(K \Delta)^{2}\right) \tau\right] \\
& =\frac{1}{2} \sqrt{\frac{\pi}{2}} e^{-\Omega_{0}^{2} / 2}\left\{K \Delta-\Omega_{0} \Omega_{1}(K \Delta)^{2}+\left[\left(\Omega_{0}^{2}-1\right)\left(\frac{\Omega_{1}^{2}}{2}+\frac{1}{8}\right)-\Omega_{0} \Omega_{2}\right](K \Delta)^{3}\right\}, \\
& \Delta \sin \left[\left(\Omega_{0}+\Omega_{1}(K \Delta)+\Omega_{2}(K \Delta)^{2}\right) \tau\right] \\
& =-\frac{\Omega_{0}}{2} K \Delta+\left[\frac{\Omega_{0}}{3} \sqrt{\frac{2}{\pi}}\left(1-e^{-\Omega_{0}{ }^{2} / 2}\right)-\frac{\Omega_{1}}{2}\right](K \Delta)^{2} \\
& +\left\{\frac{\Omega_{0}}{8}+\frac{\Omega_{1}}{3} \sqrt{\frac{2}{\pi}}\left[1+\left(\Omega_{0}^{2}-1\right) e^{-\Omega_{0}^{2} / 2}\right]-\frac{\Omega_{2}}{2}\right\}(K \Delta)^{3},
\end{aligned}
$$

where Eqs. (19) and the Gaussian distribution $g(\omega)$ have been used. After a tedious calculation, we obtain from Eq. (A.2)

$$
\begin{aligned}
\Omega_{0} \tau= & -\tan ^{-1}\left(\sqrt{\frac{2}{\pi}} \Omega_{0} e^{\Omega_{0}^{2} / 2}\right) \\
\Omega_{1} \tau= & \sqrt{\frac{8}{\pi}} e^{\Omega_{0}^{2} / 2}\left(1+\frac{2}{\pi} \Omega_{0}^{2} e^{\Omega_{0}^{2}}\right)^{-1}\left[\sqrt{\frac{2}{9 \pi}} \Omega_{0}\left(1-e^{-\Omega_{0}^{2} / 2}\right)-\frac{\Omega_{1}}{2}-\frac{\Omega_{0}^{2} \Omega_{1}}{2}\right], \\
\Omega_{2} \tau= & \sqrt{\frac{2}{\pi}} e^{-\Omega_{0}^{2} / 2}\left(1+\frac{2}{\pi} \Omega_{0}^{2} e^{\Omega_{0}^{2}}\right)^{-1}\left\{\left(1+\Omega_{0}^{2}\right)\left(\frac{\Omega_{0}}{8}-\Omega_{2}\right)\right. \\
& +\frac{8}{\pi} \Omega_{0} e^{\Omega_{0}^{2} / 2}\left(1+\frac{2}{\pi} \Omega_{0}^{2} e^{\Omega_{0}^{2}}\right)^{-1}\left[\sqrt{\frac{2}{9 \pi}} \Omega_{0}\left(1-e^{-\Omega_{0}^{2} / 2}\right)-\frac{\Omega_{1}}{2}-\frac{\Omega_{0}^{2} \Omega_{1}}{2}\right]^{2} \\
& \left.-\frac{1}{2} \Omega_{0} \Omega_{1}^{2}\left(\Omega_{0}^{2}+3\right)+\sqrt{\frac{8}{9 \pi}} \Omega_{1}\left(1+\Omega_{0}^{2}-e^{-\Omega_{0}^{2} / 2}\right)\right\}
\end{aligned}
$$

together with

$$
\Delta=a_{1} K \Delta+b_{1}(K \Delta)^{2}+c_{1}(K \Delta)^{3}+\mathcal{O}(K \Delta)^{4},
$$

which is just Eq. (20). The coefficients depend on $\Omega_{0}, \Omega_{1}$, and $\Omega_{2}$ according to

$$
\begin{aligned}
& a_{1}=\left(\frac{\Omega_{0}^{2}}{4}+\frac{\pi}{8} e^{-\Omega_{0}^{2}}\right)^{-1 / 2}, \\
& b_{1}=\left(\Omega_{0}^{2}+\frac{\pi}{2} e^{-\Omega_{0}^{2}}\right)^{-1 / 2}\left[-\sqrt{\frac{2}{9 \pi}} \Omega_{0}^{2}\left(1-e^{-\Omega_{0}^{2} / 2}\right)+\frac{\Omega_{0} \Omega_{1}}{2}\left(1-\frac{\pi}{2} e^{-\Omega_{0}^{2}}\right)\right], \\
& c_{1}=\left(\Omega_{0}^{2}+\frac{\pi}{2} e^{-\Omega_{0}^{2}}\right)^{-1 / 2}\left\{\left(\frac{2}{9 \pi}-\frac{1}{8}\right) \Omega_{0}^{2}+\frac{\Omega_{0} \Omega_{1}}{2}+\frac{\Omega_{1}^{2}}{4}-\sqrt{\frac{2}{9 \pi}} \Omega_{0} \Omega_{1}\left(2+\Omega_{0}^{2}\right)\right. \\
&-\left(\Omega_{0}^{2}+\frac{\pi}{2} e^{-\Omega_{0}^{2}}\right)^{-1}\left[\sqrt{\frac{2}{9 \pi}} \Omega_{0}^{2}\left(1-e^{-\Omega_{0}^{2} / 2}\right)-\frac{\Omega_{0} \Omega_{1}}{2}\left(1-\frac{\pi}{2} e^{-\Omega_{0}^{2}}\right)\right]^{2} \\
&-\left(\frac{4}{9 \pi} \Omega_{0}^{2}-\sqrt{\frac{8}{9 \pi}} \Omega_{0} \Omega_{1}\right) e^{-\Omega_{0}^{2} / 2} \\
&\left.+\left[\frac{2}{9 \pi} \Omega_{0}^{2}+\frac{\pi}{4}\left(\frac{\Omega_{0}^{2}}{8}-\frac{1}{8}+\Omega_{0}^{2} \Omega_{1}^{2}-\frac{\Omega_{1}^{2}}{2}-\Omega_{0} \Omega_{2}\right)\right] e^{-\Omega_{0}^{2}}\right\} .
\end{aligned}
$$

\section{APPENDIX B}

In the case $\Omega \gg K \Delta(\ll 1)$, we approximate the integral in Eq. 16 as follows: 


$$
\begin{aligned}
& \int_{1}^{\infty} d x g(\Omega+K \Delta x)\left(x-\sqrt{x^{2}-1}\right)+\int_{-\infty}^{-1} d x g(\Omega+K \Delta x)\left(x+\sqrt{x^{2}-1}\right) \\
& =\int_{1}^{\infty} d x[g(\Omega+K \Delta x)-g(\Omega-K \Delta x)]\left(x-\sqrt{x^{2}-1}\right) \\
& \approx-\int_{1}^{\infty} d x g(\Omega-K \Delta x)\left(x-\sqrt{x^{2}-1}\right),
\end{aligned}
$$

which, upon expansion to the order $(K \Delta)^{3}$, gives Eq. (16) in the form

$$
\begin{aligned}
\Delta & \cos \left[\left(\Omega_{0}+\Omega_{1}(K \Delta)+\Omega_{2}(K \Delta)^{2}\right) \tau\right] \\
= & \frac{1}{2} \sqrt{\frac{\pi}{2}} e^{-\Omega_{0}{ }^{2} / 2}\left\{K \Delta-\Omega_{0} \Omega_{1}(K \Delta)^{2}+\left[\left(\Omega_{0}{ }^{2}-1\right)\left(\frac{\Omega_{1}{ }^{2}}{2}+\frac{1}{8}\right)-\Omega_{0} \Omega_{2}\right](K \Delta)^{3}\right\}, \\
\Delta & \sin \left[\left(\Omega_{0}+\Omega_{1}(K \Delta)+\Omega_{2}(K \Delta)^{2}\right) \tau\right] \\
= & -\frac{\left(1+\Omega_{0}{ }^{2}\right)}{4 \Omega_{0}{ }^{3}}\left[1+\Phi\left(\Omega_{0} / \sqrt{2}\right)\right] K \Delta \\
+ & \frac{1}{12 \Omega_{0}{ }^{4}}\left\{-\sqrt{\frac{2}{\pi}} e^{-\Omega_{0}{ }^{2} / 2}\left(4 \Omega_{0}{ }^{5}+3 \Omega_{0}{ }^{3}+3 \Omega_{0} \Omega_{1}\right)+3 \Omega_{1}\left(3+\Omega_{0}{ }^{2}\right)\left[1+\Phi\left(\Omega_{0} / \sqrt{2}\right)\right]\right\}(K \Delta)^{2} \\
+ & \frac{1}{48 \Omega_{0}{ }^{5}}\left\{\sqrt { \frac { 2 } { \pi } } e ^ { - \Omega _ { 0 } { } ^ { 2 } / 2 } \left[16 \Omega_{0}{ }^{7} \Omega_{1}+2 \Omega_{0}{ }^{5}\left(3-14 \Omega_{1}+3 \Omega_{1}{ }^{2}\right)-12 \Omega_{0}{ }^{4} \Omega_{2}\right.\right. \\
& \left.+6 \Omega_{0}{ }^{3}\left(-1-2 \Omega_{1}+3 \Omega_{1}{ }^{2}\right)+9 \Omega_{0}\left(1+4 \Omega_{1}{ }^{2}\right)-12 \Omega_{0}{ }^{2} \Omega_{2}\right] \\
& \left.-3\left[6+24 \Omega_{1}{ }^{2}+\Omega_{0}{ }^{2}\left(1+4 \Omega_{1}{ }^{2}\right)-12 \Omega_{0} \Omega_{2}-4 \Omega_{0}{ }^{3} \Omega_{2}\right]\left[1+\Phi\left(\Omega_{0} / \sqrt{2}\right)\right]\right\}(K \Delta)^{3}
\end{aligned}
$$

with the error function $\Phi(y) \equiv(2 / \sqrt{\pi}) \int_{0}^{y} d z e^{-z^{2}}$. After a tedious calculation, we obtain from Eq. (B.2):

$$
\begin{aligned}
\Omega_{0} \tau=- & \tan ^{-1}\left\{\frac{1}{\sqrt{2 \pi}}\left(\frac{1+\Omega_{0}^{2}}{\Omega_{0}^{3}}\right) e^{\Omega_{0}^{2} / 2}\left[1+\Phi\left(\Omega_{0} / \sqrt{2}\right)\right]\right\}+\pi \\
\Omega_{1} \tau=\{1 & \left.+\frac{1}{2 \pi} \frac{\left(1+\Omega_{0}^{2}\right)^{2}}{\Omega_{0}^{6}} e^{\Omega_{0}^{2}}\left[1+\Phi\left(\Omega_{0} / \sqrt{2}\right)\right]^{2}\right\}^{-1} \\
& \times\left\{-\frac{4 \Omega_{0}}{3 \pi}-\frac{\Omega_{1}-1}{\pi \Omega_{0}}-\frac{\Omega_{1}}{\pi \Omega_{0}^{3}}+\frac{1}{\sqrt{2 \pi}} \frac{\Omega_{1}}{\Omega_{0}^{4}}\left(3-\Omega_{0}^{4}\right) e^{\Omega_{0}^{2} / 2}\left[1+\Phi\left(\Omega_{0} / \sqrt{2}\right)\right]\right\}, \\
\Omega_{2} \tau=\{ & \left.\frac{1}{\sqrt{2 \pi}}\left(\frac{1+\Omega_{0}^{2}}{\Omega_{0}^{3}}\right) e^{\Omega_{0}^{2} / 2}\left[1+\Phi\left(\Omega_{0} / \sqrt{2}\right)\right]\right\}\left\{1+\frac{1}{2 \pi} \frac{\left(1+\Omega_{0}^{2}\right)^{2}}{\Omega_{0}^{6}} e^{\Omega_{0}^{2}}\left[1+\Phi\left(\Omega_{0} / \sqrt{2}\right)\right]^{2}\right\}^{-2} \\
& \times\left\{-\frac{4 \Omega_{0}}{3 \pi}-\frac{\Omega_{1}-1}{\pi \Omega_{0}}-\frac{\Omega_{1}}{\pi \Omega_{0}^{3}}+\frac{1}{\sqrt{2 \pi}} \frac{\Omega_{1}}{\Omega_{0}^{4}}\left(3-\Omega_{0}^{4}\right) e^{\Omega_{0}^{2} / 2}\left[1+\Phi\left(\Omega_{0} / \sqrt{2}\right)\right]\right\} \\
& -\left\{\frac{1}{\sqrt{2 \pi} \Omega_{0}^{3}}\left[\frac{1}{8}\left(1-\Omega_{0}^{4}\right)-\frac{\Omega_{1}^{2}}{2}\left(5-\Omega_{0}^{4}\right)+\Omega_{0} \Omega_{2}\left(1+\Omega_{0}^{2}\right)\right] e^{\Omega_{0}^{2} / 2}\left[1+\Phi\left(\Omega_{0} / \sqrt{2}\right)\right]\right. \\
& +\frac{1}{12 \pi \Omega_{0}^{4}}\left[16 \Omega_{0}^{6} \Omega_{1}+2 \Omega_{0}^{4}\left(3-14 \Omega_{1}+3 \Omega_{1}^{2}\right)-12 \Omega_{0}^{3} \Omega_{2}-6 \Omega_{0}^{2}\left(1+2 \Omega_{1}-3 \Omega_{1}^{2}\right)\right. \\
& \left.-12 \Omega_{0} \Omega_{2}+9\left(1+4 \Omega_{1}^{2}\right)\right]-\frac{1}{4 \sqrt{2 \pi} \Omega_{0}^{5}}\left[6+24 \Omega_{1}^{2}+\Omega_{0}^{2}\left(1+4 \Omega_{1}^{2}\right)-12 \Omega_{0} \Omega_{2}-4 \Omega_{0}^{3} \Omega_{2}\right] \\
& \left.\times e^{\Omega_{0}^{2} / 2}\left[1+\Phi\left(\Omega_{0} / \sqrt{2}\right)\right]\right\}
\end{aligned}
$$

and

$$
\Delta=a_{2} K \Delta+b_{2}(K \Delta)^{2}+c_{2}(K \Delta)^{3}+\mathcal{O}(K \Delta)^{4},
$$

which is Eq. 21). Again the coefficients depend on $\Omega_{0}, \Omega_{1}$, and $\Omega_{2}$ via

$$
a_{2}=\left\{\frac{\pi}{8} e^{-\Omega_{0}^{2}}+\frac{\left(1+\Omega_{0}^{2}\right)^{2}}{16 \Omega_{0}^{6}}\left[1+\Phi\left(\Omega_{0} / \sqrt{2}\right)\right]^{2}\right\}^{-1 / 2},
$$




$$
\begin{aligned}
b_{2}=\{ & \left.\frac{\pi}{2} e^{-\Omega_{0}^{2}}+\frac{\left(1+\Omega_{0}^{2}\right)^{2}}{4 \Omega_{0}^{6}}\left[1+\Phi\left(\Omega_{0} / \sqrt{2}\right)\right]^{2}\right\}^{-1 / 2}\left\{-\frac{\pi}{4} \Omega_{0} \Omega_{1} e^{-\Omega_{0}^{2}}+\frac{1+\Omega_{0}^{2}}{8 \Omega_{0}^{6}}\left[1+\Phi\left(\Omega_{0} / \sqrt{2}\right)\right]\right. \\
& \left.\times\left[\frac{2}{\pi} e^{-\Omega_{0}^{2} / 2}\left(\frac{4}{3} \Omega_{0}^{4}+\Omega_{0}^{2}\left(\Omega_{1}-1\right)+\Omega_{1}\right)-\left(3+\Omega_{0}^{2}\right) \Omega_{1}\left[1+\Phi\left(\Omega_{0} / \sqrt{2}\right)\right]\right]\right\} \\
c_{2}=\{ & \left.\frac{\pi}{2} e^{-\Omega_{0}^{2}}+\frac{\left(1+\Omega_{0}^{2}\right)^{2}}{4 \Omega_{0}^{6}}\left[1+\Phi\left(\Omega_{0} / \sqrt{2}\right)\right]^{2}\right\}^{-3 / 2}\left\{-\frac{\pi}{4} \Omega_{0} \Omega_{1} e^{-\Omega_{0}^{2}}+\frac{1+\Omega_{0}^{2}}{8 \Omega_{0}^{6}}\left[1+\Phi\left(\Omega_{0} / \sqrt{2}\right)\right]\right. \\
& \left.\times\left[\sqrt{\frac{2}{\pi}} e^{-\Omega_{0}^{2} / 2}\left(\frac{4}{3} \Omega_{0}^{4}+\Omega_{0}^{2}\left(\Omega_{1}-1\right)+\Omega_{1}\right)-\left(3+\Omega_{0}^{2}\right) \Omega_{1}\left[1+\Phi\left(\Omega_{0} / \sqrt{2}\right)\right]\right]\right\}^{2} \\
& +\left\{\frac{\pi}{2} e^{-\Omega_{0}^{2}}+\frac{\left(1+\Omega_{0}^{2}\right)^{2}}{4 \Omega_{0}^{6}}\left[1+\Phi\left(\Omega_{0} / \sqrt{2}\right)\right]^{2}\right\}^{-1 / 2}\left\{\frac{\pi}{8} e^{-\Omega_{0}^{2}}\left(2 \Omega_{0}^{2} \Omega_{1}^{2}+\frac{\Omega_{0}^{2}}{4}-\Omega_{1}^{2}-2 \Omega_{0} \Omega_{2}-\frac{1}{4}\right)\right. \\
& +\frac{1}{144 \Omega_{0}^{7}}\left[\sqrt{\frac{2}{\pi}} e^{-\Omega_{0}^{2} / 2}\left(\frac{4}{3} \Omega_{0}^{4}+\Omega_{0}^{2}\left(\Omega_{1}-1\right)+\Omega_{1}\right)-\left(3+\Omega_{0}^{2}\right) \Omega_{1}\left[1+\Phi\left(\Omega_{0} / \sqrt{2}\right)\right]\right]^{2} \\
& -\sqrt{\frac{2}{\pi}} e^{-\Omega_{0}^{2} / 2} \frac{1+\Omega_{0}^{2}}{96 \Omega_{0}^{7}}\left[16 \Omega_{0}^{6} \Omega_{1}+2 \Omega_{0}^{4}\left(3-14 \Omega_{1}+3 \Omega_{1}^{2}\right)-12 \Omega_{0}^{3} \Omega_{2}\right. \\
& \left.-6 \Omega_{0}^{2}\left(1+2 \Omega_{1}-3 \Omega_{1}^{2}\right)-12 \Omega_{0} \Omega_{2}+9\left(1+4 \Omega_{1}^{2}\right)\right]\left[1+\Phi\left(\Omega_{0} / \sqrt{2}\right)\right] \\
& \left.-\frac{1}{4 \sqrt{2 \pi} \Omega_{0}^{5}}\left[6+24 \Omega_{1}^{2}+\Omega_{0}^{2}\left(1+4 \Omega_{1}^{2}\right)-12 \Omega_{0} \Omega_{2}-4 \Omega_{0}^{3} \Omega_{2}\right]\right\} .
\end{aligned}
$$


[1] For a list of references, see A. T. Winfree, The Geometry of Biological Time (Springer-Verlag, New York, 1980); Y. Kuramoto, Chemical Oscillations, Waves, and Turbulence (Springer-Verlag, Berlin, 1984); L. Glass and M. C. Mackey, From Clocks to Chaos: The Rhythm of Life (Princeton Univ., Princeton, 1988).

[2] T. J. Walker, Science 166, 891 (1969); M. K. McClintock, Nature 229, 244 (1971); D. C. Michaels, E. P. Maytyas, and J. Jalife, Circ. Res. 61, 704 (1987); J. Buck, Q. Rev. Biol. 63, 265 (1988); R. D. Traub, R. Miles, and R. K. S. Wong, Science 243, 1319 (1989).

[3] R. Eckhorn et al., Biol. Cybern. 60, 121 (1988); C. M. Gray and W. Singer, Proc. Natl. Sci. USA 86, 1698 (1989).

[4] S. P. Benz, M. Rzchowski, M. Tinkham, and C. J. Lobb, Phys. Rev. Lett. 64, 693 (1990); H. C. Lee, et al., Phys. Rev. B 44, 921 (1991).

[5] Y. Kuramoto, in Proceedings of the International Symposium on Mathematical Problems in Theoretical Physics, edited by H. Araki (Springer-Verlag, New York, 1975); Y. Kuramoto and I. Nishikawa, J. Stat. Phys. 49, 569 (1987); Y. Kuramoto, Physica D 50, 15 (1991).

[6] H. Daido, Prog. Theor. Phys. 77, 622 (1987); Phys. Rev. Lett. 68, 1073 (1992).

[7] S. H. Strogatz, C. M. Marcus, R. M. Westervelt, and R. E. Mirollo, Physica D 36, 23 (1989); J. W. Swift, S. H. Strogatz, and K. Wiesenfeld, ibid. 55, 239 (1992); S. H. Strogatz, R. E. Mirollo, and P. C. Matthews, Phys. Rev. Lett. 68, 2730 (1992); S. Watanabe and S. H. Strogatz, ibid. 70, 2391 (1993); K. Wiesenfeld and J. W. Swift, Phys. Rev. E 51, 1020 (1995); K. Wiesenfeld, P. Colet, and S. H. Strogatz, Phys. Rev. Lett. 76, 404 (1996).

[8] M. Y. Choi, Phys. Rev. B 46, 564 (1992); K. Park and M. Y. Choi, Phys. Rev. B 56, 387 (1997).

[9] M. Y. Choi, Y. W. Kim, and D. C. Hong, Phys. Rev E 49, 3825 (1994); H. Hong, M. Y. Choi, J. Yi, and K.-S. Soh, ibid. 59, 353 (1999).

[10] A. Arenas and C. J. Perez Vicente, Europhys. Lett. 26, 79 (1994); K. Park and M. Y. Choi, Phys. Rev. E 52, 2907 (1995); K. Park, S. W. Rhee, and M.Y. Choi, ibid. 57, 5030 (1998).

[11] H. Tanaka, A. J. Lichtenberg, and S. Oishi, Phys. Rev. Lett. 78, 2104 (1997); H. Hong, M. Y. Choi, B.-G. Yoon, K. Park, and K.-S. Soh, J. Phys. A 32, L9 (1999).

[12] M. Y. Choi and B. A. Huberman, Phys. Rev. B 31, 2861 (1985).

[13] H. G. Shuster and P. Wagner, Prog. Theor. Phys. 81, 939 (1989).

[14] T. B. Luzyanina, Network, 6, 43 (1995).

[15] E. Niebur, H. G. Schuster, and D. M. Kammen, Phys. Rev. Lett. 67, 2753 (1991).

[16] Y. Nakamura, F. Tominaga, and T. Munakata, Phys. Rev. E 49, 4849 (1994); S. Kim, S. H. Park, and C. S. Ryu, Phys. Rev. Lett. 79, 2911 (1997).

[17] M. K. S. Yeung and S. H. Strogatz, Phys. Rev. Lett. 82, 648 (1999).

[18] H. Risken, The Fokker-Planck Equation: Methods of Solution and Applications (Springer-Verlag, Berlin, 1989).

[19] H. Hong, M. Y. Choi, K. Park, B.-G. Yoon, and K.-S. Soh, Phys. Rev. E (in press). 


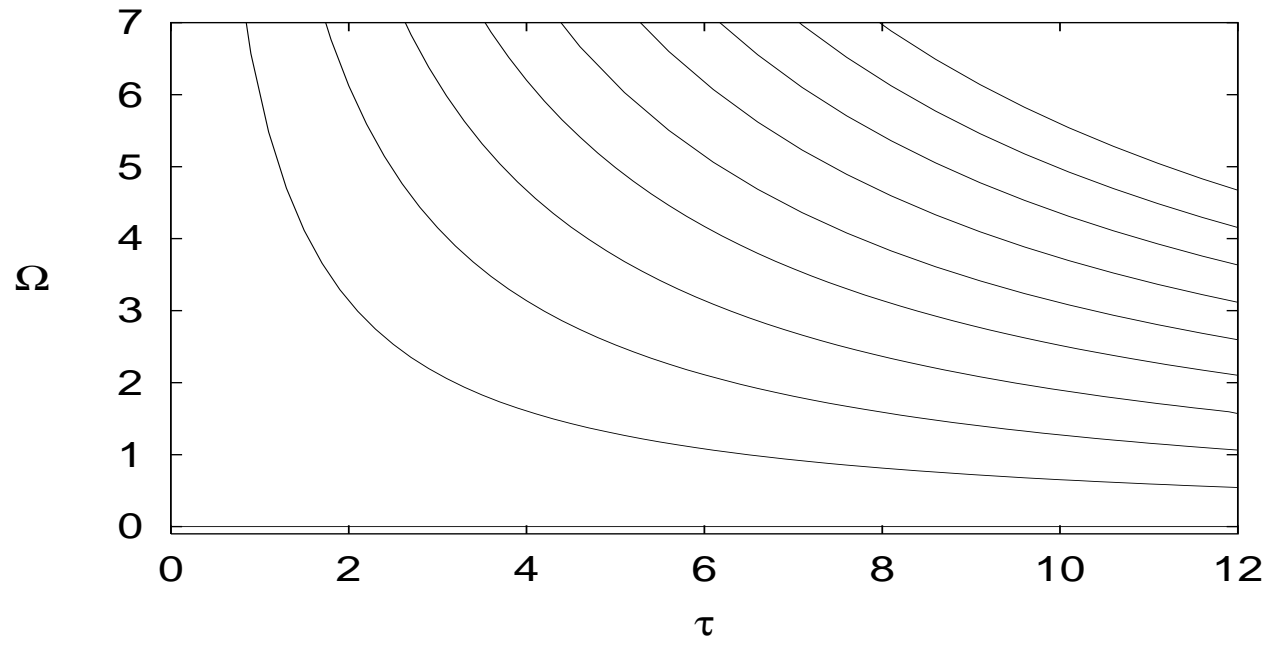

(a)

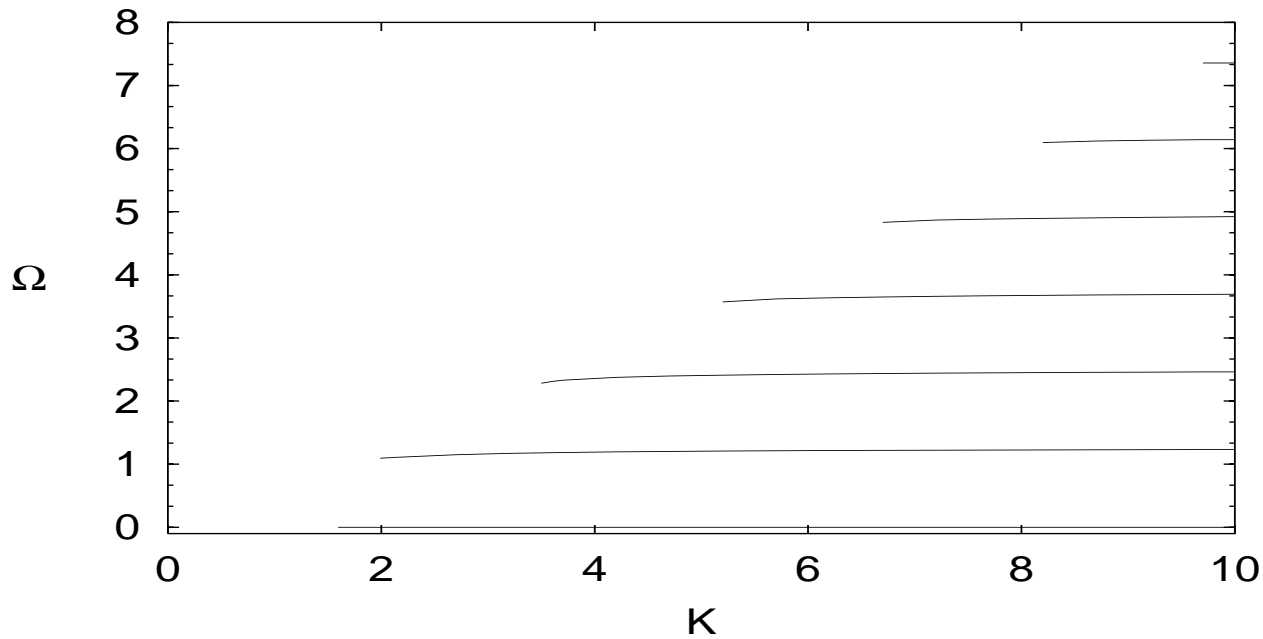

(b)

FIG. 1. Synchronization frequency $\Omega$ (a) as functions of the delay time $\tau$ at the coupling strength $K=10$, where frequency suppression and multistability can be observed; (b) as functions of $K$ at $\tau=5$. For given $K$, the largest synchronization frequency belonging to the highest stair gives the most stable solution, but the corresponding basin of attraction is small. 


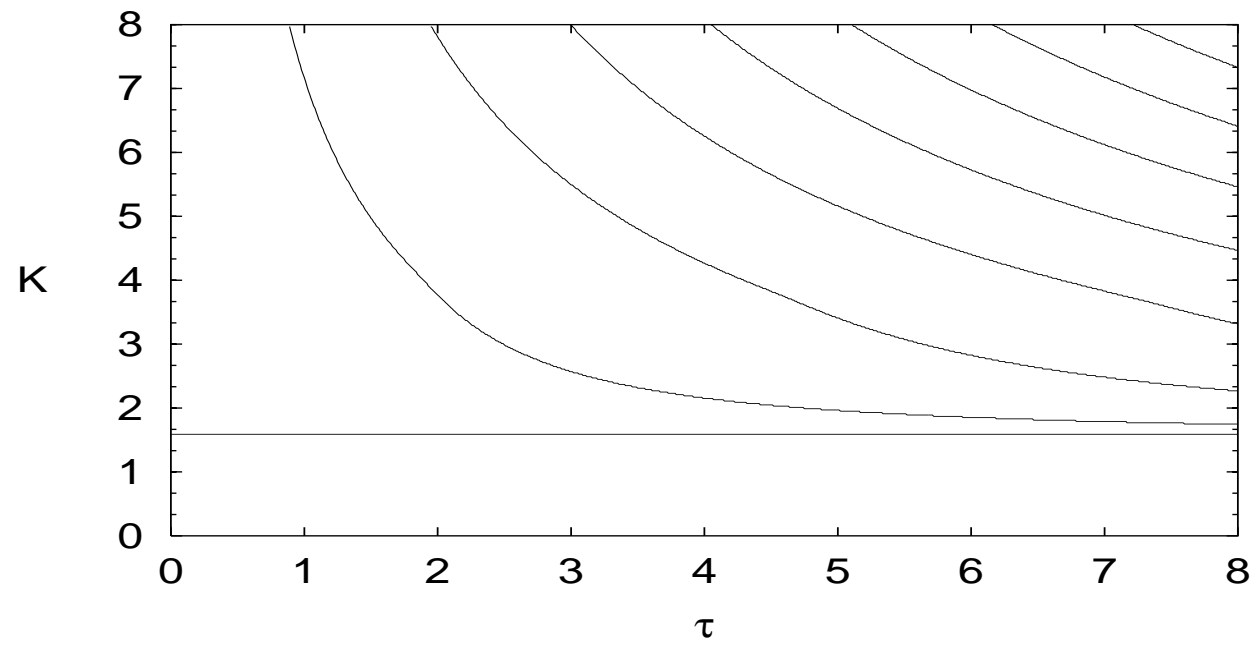

(a)

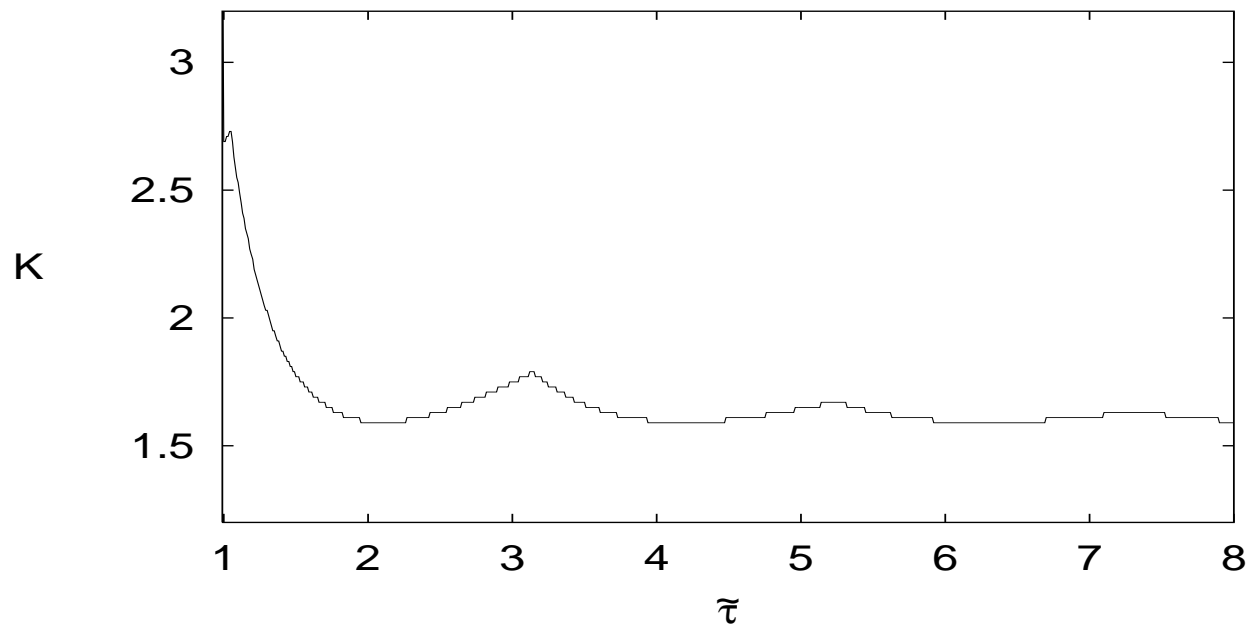

(b)

FIG. 2. Phase diagram on the $K-\tau$ plane, displaying boundaries between the incoherent and coherent states. Whenever each boundary in (a) is crossed from below, a new additional coherent state with a larger synchronization frequency emerges. Below the lowest boundary, which is the straight line $K=K_{c} \approx 1.596$, only the incoherent state exists. In (b) the boundaries are redrawn, with the horizontal axis rescaled: $\tilde{\tau}$ in (b) corresponds to $\Omega \tau /(\Omega+3)$ in (a). Here displayed is only the envelope consisting of the curve segments with lowest values of $K$ at given $\tilde{\tau}$, which describes the phase boundary separating the incoherent state in the system with $\omega_{0}=3$. 


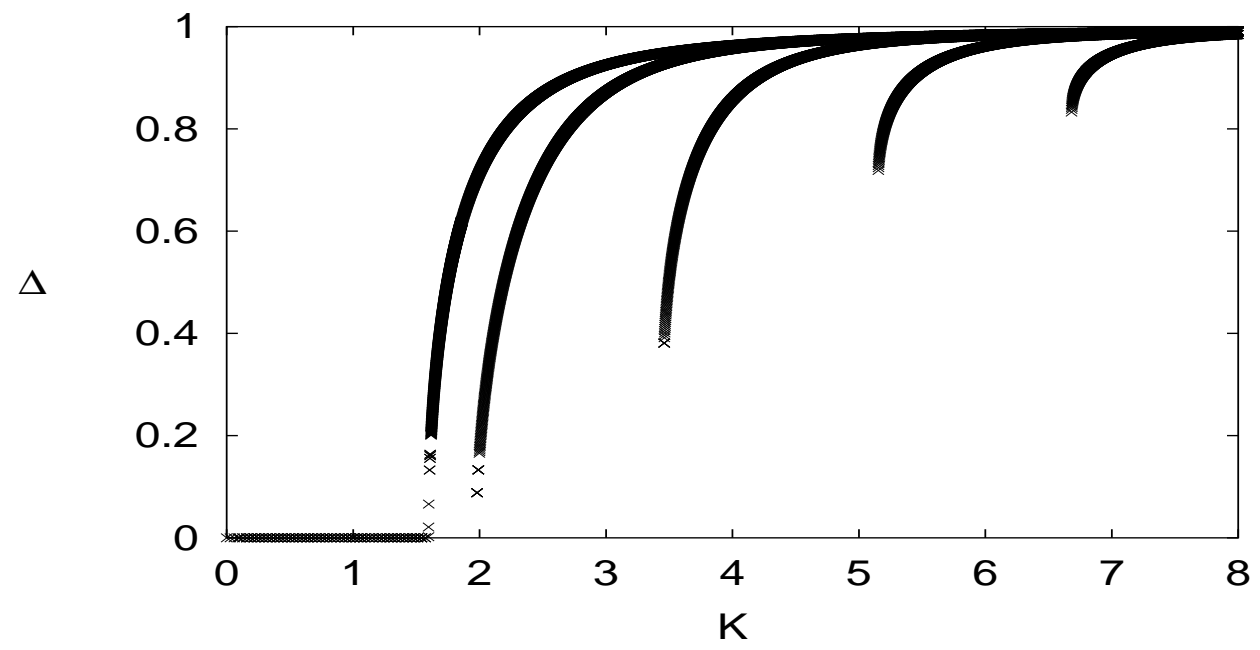

FIG. 3. Order parameter $\Delta$ as a function of the coupling strength $K$ at the time delay $\tau=5$. Each line describes the transition for each synchronization frequency, and the critical coupling strength $K_{c}$ is shown to increase for large $\Omega$. The leftmost curve, which corresponds to $\Omega=0$, gives $K_{c} \approx 1.596$; the next one, corresponding to $\Omega \approx 1.09$, gives $K_{c} \approx 1.97$. The numerical results displayed by the leftmost curve agree well with the analytical ones.

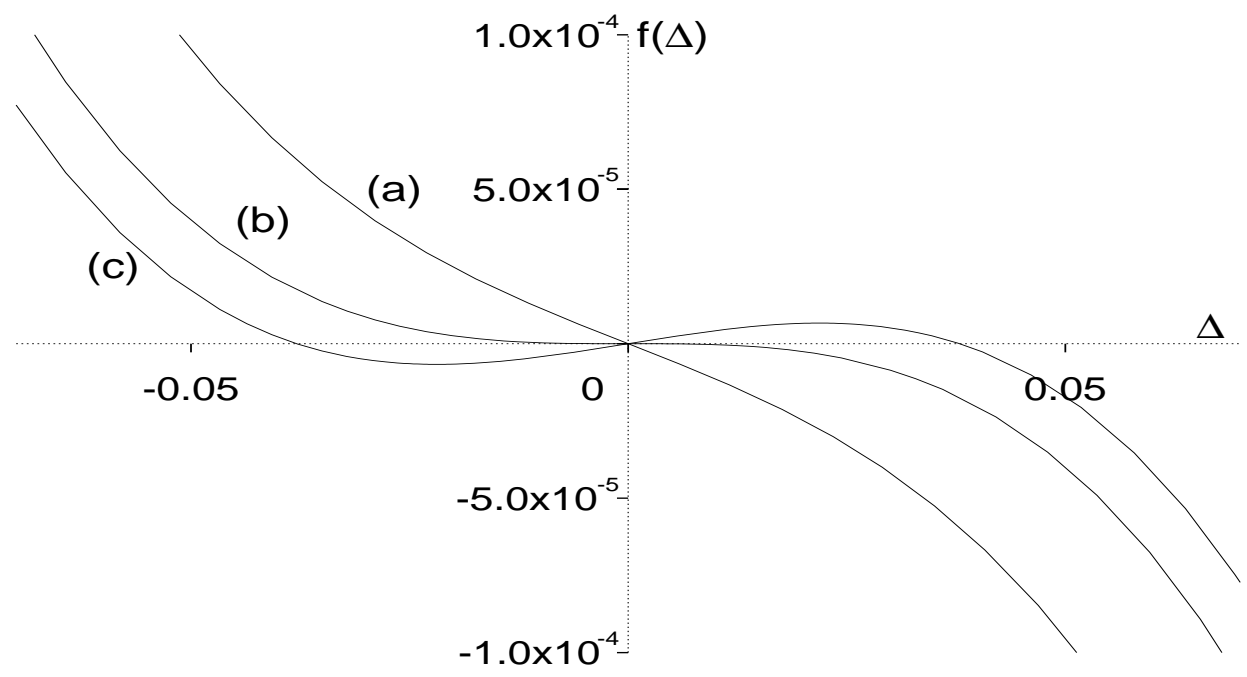

FIG. 4. Graphical solutions of Eq. 20, displaying $f(\Delta) \equiv\left(a_{1} K-1\right) \Delta+b_{1}(K \Delta)^{2}+c_{1}(K \Delta)^{3}$ versus $\Delta$, for $b_{1}=0$ with (a) $K=1.594\left(<K_{c}\right)$, (b) $K=1.596\left(=K_{c}\right)$, and (c) $K=1.597\left(>K_{c}\right)$. The negative solution appearing in (c) is unphysical. 


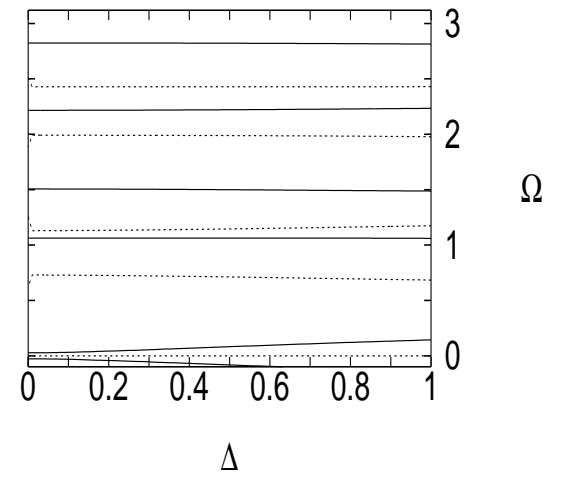

(a)

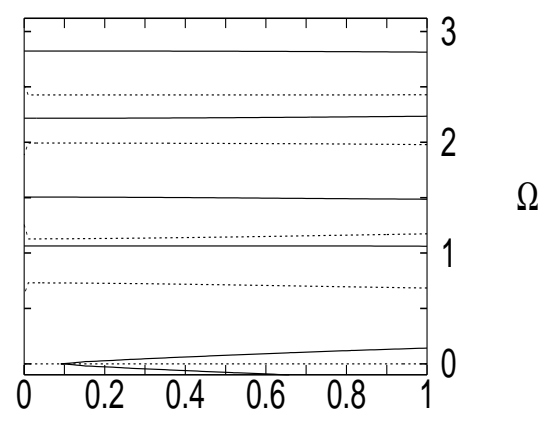

$\Delta$

$(c)$

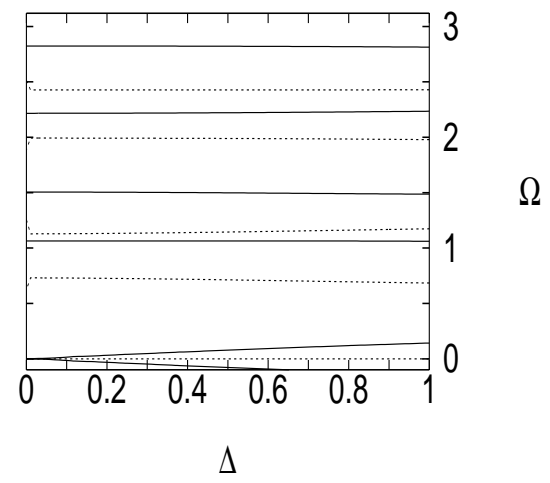

(b)

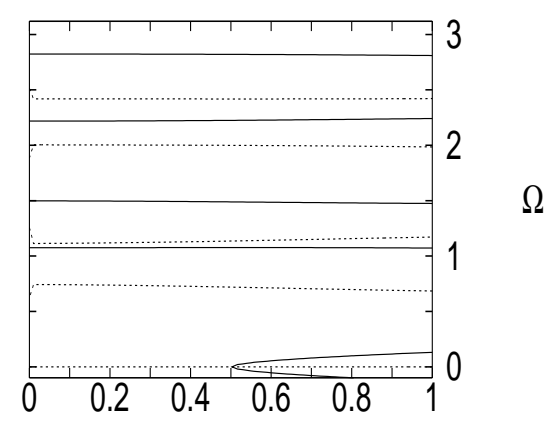

$\Delta$

(d)

FIG. 5. Synchronization frequency $\Omega$ versus the order parameter $\Delta$ obtained from Eq. (16) for $\tau=5$ at (a) $K=1.58$, (b) $K=1.596$, (c) $K=1.60$, (d) $K=1.75$. Solid and dotted lines represent solutions of the first equation and the second equation of Eq. (16). A continuous transition for $\Omega=0$ can be observed at $K=1.596$. 


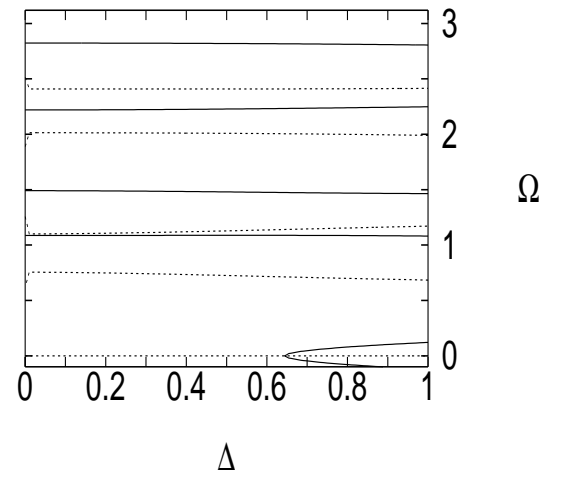

(a)

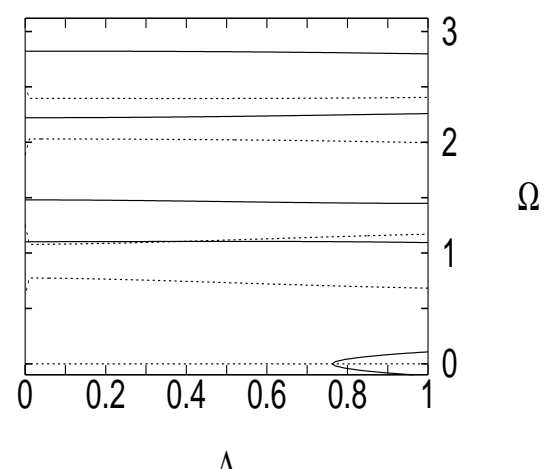

(c)

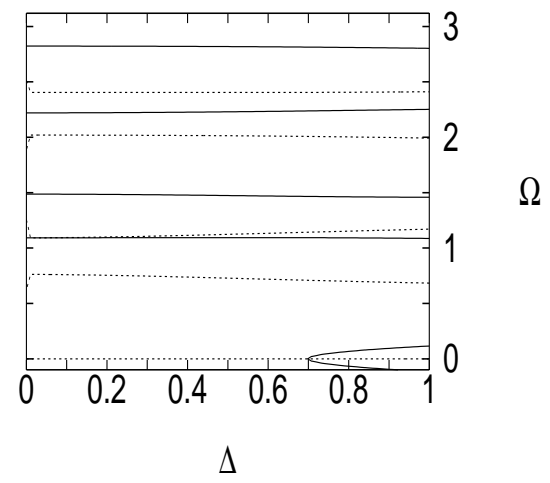

(b)

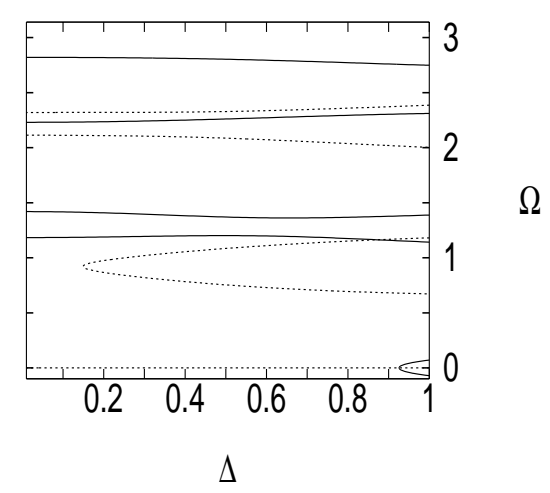

$(d)$

FIG. 6. Synchronization frequency $\Omega$ versus the order parameter $\Delta$ obtained from Eq. (16) for $\tau=5$ at (a) $K=1.89$, (b) $K=1.97$, (c) $K=2.10$, (d) $K=3.00$. A discontinuous transition for $\Omega \approx 1.09$ can be observed at $K=1.97$. 


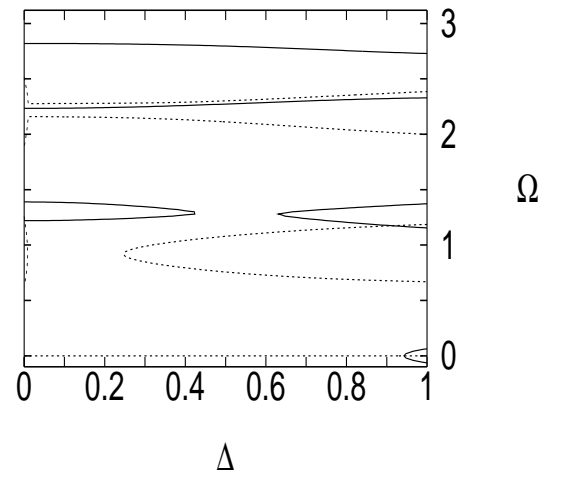

(a)

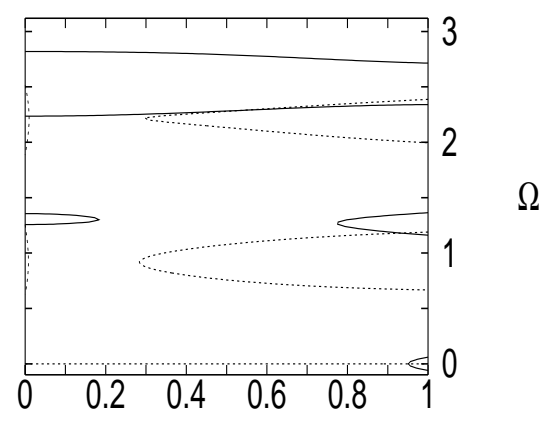

$\Delta$

$(c)$

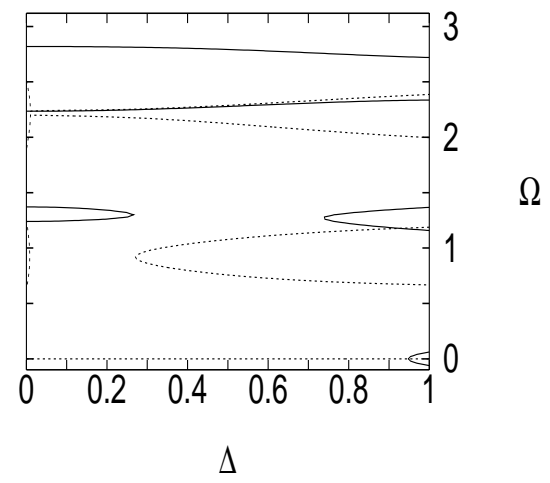

(b)

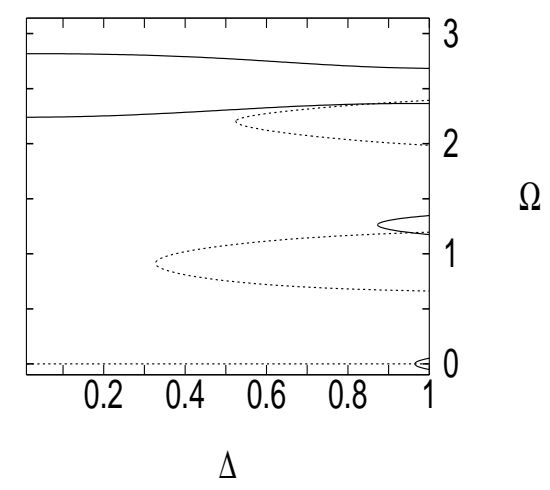

(d)

FIG. 7. Synchronization frequency $\Omega$ versus the order parameter $\Delta$ obtained from Eq. (16) for $\tau=5$ at (a) $K=3.30$, (b) $K=3.46$, (c) $K=3.515$, (d) $K=4.00$. A discontinuous transition for $\Omega \approx 2.25$ can be observed at $K=3.46$. 


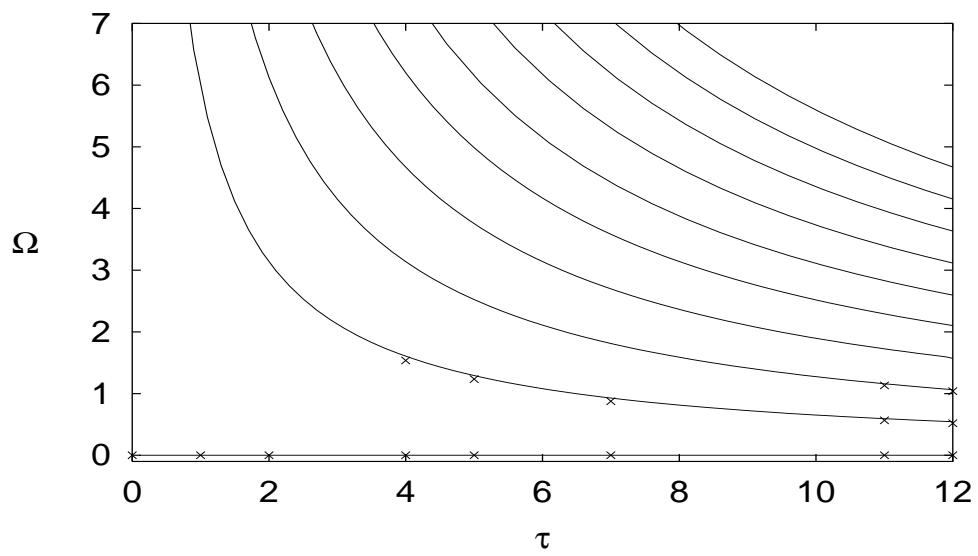

(a)

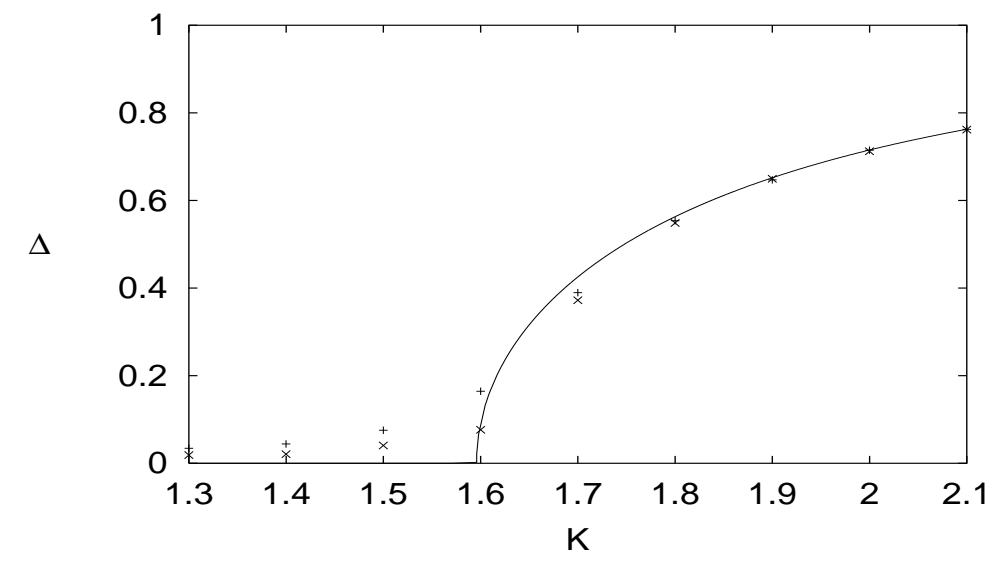

(b)

FIG. 8. Results of numerical simulations on 5000 coupled oscillators: (a) Synchronization frequency versus the delay time. Crosses are results of numerical simulations and solid lines represent the solutions of Eq. (16), displaying perfect agreement with each other. (b) Order parameter versus the coupling strength, displaying continuous transitions (with zero synchronization frequency) for $\tau=0$ (plus signs) and $\tau=5$ (crosses). The solid line represents analytic results for $\tau=5$, displaying reasonable agreement with the numerical ones. Slight suppression of synchronization by time delay can be observed near the transition region. The size of the error bars estimated by the standard deviation is about that of the symbols and lines are merely guides to the eye. 